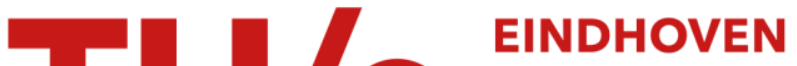

\section{A hybrid Fourier PSTD/DG method to solve the linearized Euler equations}

\section{Citation for published version (APA):}

Pagán Muñoz, R., \& Hornikx, M. (2016). A hybrid Fourier PSTD/DG method to solve the linearized Euler equations: optimization and accuracy. In 22nd AIAA/CEAS Aeroacoustics Conference, 30 May - 1 June 2016, Lyon, France [AIAA-2016-2718] American Institute of Aeronautics and Astronautics Inc. (AIAA). https://doi.org/10.2514/6.2016-2718

DOI:

10.2514/6.2016-2718

Document status and date:

Published: 01/05/2016

\section{Document Version:}

Publisher's PDF, also known as Version of Record (includes final page, issue and volume numbers)

\section{Please check the document version of this publication:}

- A submitted manuscript is the version of the article upon submission and before peer-review. There can be important differences between the submitted version and the official published version of record. People interested in the research are advised to contact the author for the final version of the publication, or visit the $\mathrm{DOI}$ to the publisher's website.

- The final author version and the galley proof are versions of the publication after peer review.

- The final published version features the final layout of the paper including the volume, issue and page numbers.

Link to publication

\section{General rights}

Copyright and moral rights for the publications made accessible in the public portal are retained by the authors and/or other copyright owners and it is a condition of accessing publications that users recognise and abide by the legal requirements associated with these rights.

- Users may download and print one copy of any publication from the public portal for the purpose of private study or research.

- You may not further distribute the material or use it for any profit-making activity or commercial gain

- You may freely distribute the URL identifying the publication in the public portal.

If the publication is distributed under the terms of Article 25fa of the Dutch Copyright Act, indicated by the "Taverne" license above, please follow below link for the End User Agreement:

www.tue.nl/taverne

Take down policy

If you believe that this document breaches copyright please contact us at:

openaccess@tue.nl

providing details and we will investigate your claim. 


\title{
A hybrid Fourier PSTD/DG method to solve the linearized Euler equations: optimization and accuracy
}

\author{
Raúl Pagán Muñoz* and Maarten Hornikx ${ }^{\dagger}$ \\ Eindhoven University of Technology, Eindhoven, 5600 MB, The Netherlands
}

\begin{abstract}
The wave-based Fourier Pseudospectral time-domain (Fourier PSTD) method was shown to be an effective way of modelling acoustic propagation problems as described by the linearized Euler equations (LEE), but is limited to real-valued frequency independent boundary conditions and predominantly staircase-like boundary shapes. This paper presents a hybrid approach to solve the LEE, coupling Fourier PSTD with a nodal Discontinuous Galerkin (DG) method. DG exhibits almost no restrictions with respect to geometrical complexity or boundary conditions. The aim of this novel method is to allow the computation of arbitrary boundary conditions and complex geometries by using the benefits of DG, while keeping Fourier PSTD in the bulk of the domain. The hybridization approach is based on conformal meshes to avoid spatial interpolation of the DG solutions when transferring values from DG to Fourier PSTD and a Gaussian window function to enforce periodicity, while the data transfer from Fourier PSTD to DG is done utilizing spectral interpolation of the Fourier PSTD solutions. Furthermore, the coupling algorithm includes a low-pass filtering approach to suppress instabilities arising from the Fourier PSTD solver. In this paper, the influence of the main parameters of the data-exchange areas and the Gaussian window function on the hybrid method results, is investigated. The precision of the hybrid approach is presented and compared with the precision of the Fourier PSTD standalone solver, showing no significant additional error from the hybrid approach for a suitable selection of parameters.
\end{abstract}

\section{Nomenclature}

$N \quad$ Polynomial order, total number of points

$T \quad$ Total number of elements

$c \quad$ Sound velocity, $\mathrm{m} / \mathrm{s}$

$k \quad$ Wave number, $m^{-1}$

$n \quad$ Normal direction

$\mathrm{p}$ Pressure, $\mathrm{Pa}$

$u \quad$ Velocity, $\mathrm{m} / \mathrm{s}$

$\Delta x \quad$ Element size in one dimension, $m$

$\Delta t \quad$ Time step, $s$

$\Omega \quad$ Physical or computational domain

$\epsilon \quad$ Error, $d B$

$\nu \quad$ Courant number

$\rho \quad$ Density, $\mathrm{kg} / \mathrm{m}^{3}$

Subscript

$P S \quad$ Fourier PSTD domain

$D G$ DG domain

amp Amplitude or dissipation

$i \quad$ Variable number, imaginary number

${ }^{*}$ Doctoral candidate, Building Physics and Services, Department of the Built Environment, P.O.Box 513, 5612 AP, Eindhoven.

${ }^{\dagger}$ Assistant Professor, Building Physics and Services, Department of the Built Environment, P.O.Box 513, 5612 AP, Eindhoven. 


$\begin{array}{ll}j, l, n & \text { Variable number } \\ \lambda & \text { Wavelength, } m \\ \phi & \text { Phase or dispersion }\end{array}$

\section{Introduction}

The benefits of using high order methods when solving time dependent problems have already been documented, for instance, by Hesthaven et al. ${ }^{1}$ Among high order methods, Fourier pseudospectral techniques have shown to be an effective way of modelling wave propagation ${ }^{2}$ and, particularly, the Fourier Pseudospectral time-domain (Fourier PSTD) technique has shown to be suitable for aeroacoustics applications. ${ }^{3}$ Fourier PSTD discretizes the physical domain in a regular mesh, calculating the solutions at discrete points where the spatial variables are transformed through a set of basis functions and the derivatives are computed in the transformed domain. Since the chosen basis functions consist of (periodic) trigonometric polynomials, the domain transformation can be computed by fast Fourier transforms (FFT). The main benefit is that Fourier PSTD only requires the theoretical minimum number of points per wavelength $\left(N_{\lambda}=2\right)$ to solve the acoustic problem of interest with spectral accuracy, as shown by Liu. ${ }^{4}$ Therefore, Fourier PSTD is based on Fourier analysis and synthesis to compute the spatial derivatives of the governing equation whereas the time-marching scheme is operated by another method, e.g., the Runge-Kutta (RK) method. Hence, Fourier PSTD is an efficient algorithm to solve acoustic propagation problems but it suffers from the well-known Gibbs phenomenon when computing the derivatives of a non-smooth or discontinuous function. This major limitation for the computation of the boundaries, caused by the periodicity assumed in the FFT, can be solved, e.g., by using perfectly match layers (PML) ${ }^{5}$ when non-reflected boundaries are computed. In case of rigid boundaries and boundaries with a different density, solutions have been successfully presented, for instance by Hornikx et al., ${ }^{2}$ and one approximation for impedance boundary conditions was introduced by Spa et al. ${ }^{6}$ However, no accurate solution for frequency dependent boundary conditions has been presented thus far. Furthermore, the method is limited to predominantly staircase-like boundary shapes, like in the widely used finite-difference time-domain method (FDTD). ${ }^{7}$ Although, a successful development has been presented by Hornikx et al. ${ }^{8}$ to locally refine the grid using multidomain implementations, its performance is limited when computing complex geometries.

The idea of coupling methodologies in order to get the benefits of each solver has already been presented by many authors as, for instance, Utzmann et al. ${ }^{9}$ Léger et al. ${ }^{10}$ and, recently, Lisitsa et al. ${ }^{11}$ The present paper introduces a hybrid approach to solve the linearized Euler equations (LEE), coupling Fourier PSTD with the nodal Discontinuous Galerkin (DG) method. DG exhibits almost no restrictions with respect to geometrical complexity or boundary conditions, and has been successfully implemented for aeroacoustics applications, for instance. ${ }^{12-15}$ The aim of the current novel method is to allow the computation of arbitrary boundary conditions and complex geometries by using the benefits of the DG method at the boundaries while keeping Fourier PSTD in the bulk of the domain, as in the schematic example shown in figure 1 for a twodimensional domain. In particular, this would be beneficial for atmospheric sound propagation, with DG near the ground surface and Fourier PSTD in the (moving and inhomogeneous) propagation domain above it. DG is a finite element scheme that operates in a functional space of piecewise polynomial functions with no continuity constraint at cell interfaces. The cells are connected to its neighbours via numerical flux terms based on the values of the solutions at both sides of the interface. In this work, the quadraturefree DG method introduced by Atkins and $\mathrm{Shu}^{16}$ has been implemented following the algorithms for the nodal approach presented by Hesthaven and Warburton, ${ }^{17}$ and using interpolating Lagrange polynomials on a nodal set of Legendre-Gauss-Lobatto points. Finally, the DG method is combined with a low-storage RK scheme ${ }^{18}$ for time integration. DG is a local method adequate to handle complex geometries, e.g., ${ }^{15}$ it allows to locally refine the polynomial order and/or the element size, and it is well suited for parallel computing. Furthermore, boundary impedance condition formulations have already been presented, for instance by Reymen et al. ${ }^{19}$ Overall, DG is a suitable option to complement Fourier PSTD. However, in contrast with the spectral accuracy of Fourier PSTD, the dispersion and dissipation DG errors converge with order $\Delta x_{D G}^{(2 N+3)}$ and $\Delta x_{D G}^{(2 N+2)}$, respectively, for a general upwind flux (with $\Delta x_{D G}$ the element size and $\mathrm{N}$ the order of the scheme), given that more than $\pi$ points per wavelength are present. ${ }^{17}$ Due to its higher demand on the spatial discretization, DG is only used for the computation of the boundaries in the hybrid approach. 


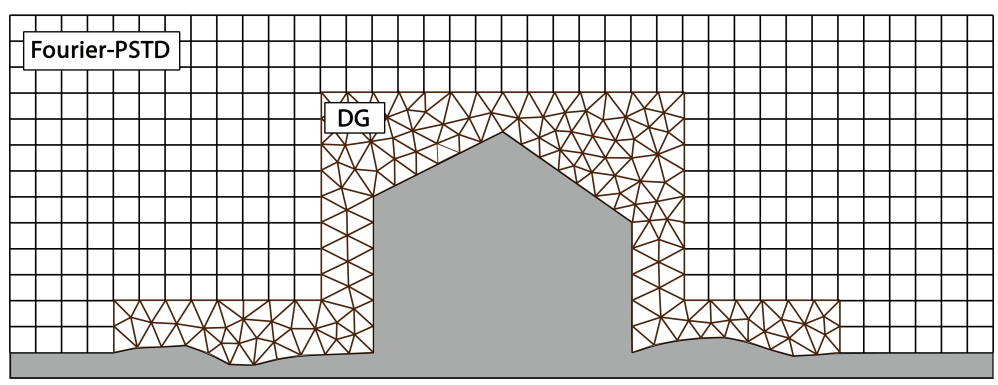

Figure 1: Schematic example of an application of the hybrid method in a two-dimensional domain.

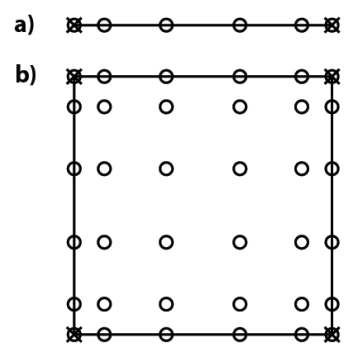

Figure 2: One overlapping element from the hybrid Fourier PSTD/DG conformal mesh for a) one-dimensional case and b) two-dimensional case (quadrilateral DG element). The figure shows the nodal distribution in DG for polynomial order $N=5$ (circles) and the Fourier PSTD nodes (crosses).

In this work, the physical domain $\Omega$ is decomposed into two computational subdomains $\left(\Omega_{D G}\right.$ and $\left.\Omega_{P S}\right)$ with an overlapping area, where the solutions of the LEE are approximated by the numerical methods. The hybrid method couples the results of both solvers in the overlapping area. The approach is based on conformal meshes, as shown in figure 2, to avoid spatial interpolation of the DG solutions when transferring values from DG to Fourier PSTD, however the interpolation of the Fourier PSTD solutions is needed before copying values to the DG solver. Furthermore, the coupling algorithm includes a Gaussian window function to impose periodicity in the Fourier PSTD solver after transferring data from DG. Therefore, due to the periodicity of the Fourier PSTD method, the solutions are updated over a zone rather than at a single point which allows to have local time stepping in each solver and the data update between them is only done after the larger (Fourier PSTD) time steps.

In this work, the error of the hybrid methodology for different parameters of the data-exchange areas and the Gaussian window function is investigated and reported. A suitable combination of these parameters is selected to compute the total error of the hybrid method for one-dimensional cases. Furthermore, the influence of the DG polynomial order in the global error is computed. The precision of the hybrid approach is presented and compared with the precision of the Fourier PSTD standalone solver. This paper is structured as follows. Section II presents the main features of the physical equations and the numerical methods. In section III, the hybridization process is detailed. Finally, the main sources of error of the hybrid method are evaluated and reported in section IV together with a comparison with the results from a Fourier PSTD standalone solver in order to establish the precision of the hybrid approach.

\section{Physical equations and numerical methods}

\section{A. The linearized Euler equations}

Acoustic propagation investigated in this paper is governed by the LEE. In three-dimensional Cartesian coordinates, the governing equation in conservative form reads: 


$$
\begin{aligned}
& \frac{\partial \mathbf{q}}{\partial t}+\mathbf{A}_{\mathbf{j}} \frac{\partial \mathbf{q}}{\partial x_{j}}+\mathbf{C q}=0, \\
& \mathbf{q}=\left[\rho, \rho_{0} u_{1}, \rho_{0} u_{2}, \rho_{0} u_{3}, p\right]^{T}, \\
& \mathbf{A}_{\mathbf{j}}=\left[\begin{array}{ccccc}
u_{0, j} & \delta_{1, j} & \delta_{2, j} & \delta_{3, j} & 0 \\
0 & u_{0, j} & 0 & 0 & \delta_{1, j} \\
0 & 0 & u_{0, j} & 0 & \delta_{2, j} \\
0 & 0 & 0 & u_{0, j} & \delta_{3, j} \\
0 & c_{0}^{2} \delta_{1, j} & c_{0}^{2} \delta_{2, j} & c_{0}^{2} \delta_{3, j} & u_{0, j}
\end{array}\right] \\
& \mathbf{C}=\left[\begin{array}{ccccc}
0 & 0 & 0 & 0 & 0 \\
u_{0, j} \frac{\partial u_{0,1}}{\partial x_{j}} & \frac{\partial u_{0,1}}{\partial x_{1}} & \frac{\partial u_{0,1}}{\partial x_{2}} & \frac{\partial u_{0,1}}{\partial x_{3}} & 0 \\
u_{0, j} \frac{\partial u_{0,2}}{\partial x_{j}} & \frac{\partial u_{0,2}}{\partial x_{1}} & \frac{\partial u_{0,2}}{\partial x_{2}} & \frac{\partial u_{0,2}}{\partial x_{3}} & 0 \\
u_{0, j} \frac{\partial u_{0,3}}{\partial x_{j}} & \frac{\partial u_{0,3}}{\partial x_{1}} & \frac{\partial u_{0,3}}{\partial x_{2}} & \frac{\partial u_{0,3}}{\partial x_{3}} & 0 \\
0 & \frac{(1-\gamma)}{\rho_{0}} \frac{\partial p_{0}}{\partial x_{1}} & \frac{(1-\gamma)}{\rho_{0}} \frac{\partial p_{0}}{\partial x_{2}} & \frac{(1-\gamma)}{\rho_{0}} \frac{\partial p_{0}}{\partial x_{3}} & (\gamma-1) \frac{\partial u_{0, j}}{\partial x_{j}}
\end{array}\right],
\end{aligned}
$$

where the acoustic variables $\mathbf{q}$ are the density $\rho$, the velocity components $u_{j}$ and the pressure $p$. The matrix $\mathbf{A}_{\mathbf{j}}$ accounts for the Jacobian flux, whereas $\mathbf{C}$ includes the non-uniform atmospheric mean flows. $\gamma$ is the heat capacity ratio and $\delta$ denotes the Kronecker delta function. The adiabatic sound speed can be calculated as $c_{0}^{2}=\gamma p_{0} / \rho_{0}$. The physical variables are decomposed into their ambient values, denoted by subscript 0 , and the acoustic fluctuations.

To simplify the model, in this work the propagation medium is at rest and its temperature is constant in space and time. Therefore, the term Cq of Eq. (1) is not included in the calculations. The LEE used in this work for one-dimensional cases are reduced to Eqs. (2) and are completed with some initial and boundary conditions.

$$
\begin{aligned}
\frac{\partial u_{x}}{\partial t}+\frac{1}{\rho_{0}} \frac{\partial p}{\partial x} & =0, \\
\frac{\partial p}{\partial t}+\rho_{0} c_{0}^{2} \frac{\partial u_{x}}{\partial x} & =0 .
\end{aligned}
$$

\section{B. Fourier PSTD method}

Fourier PSTD is a global wave-based time-domain method suitable for the computation of acoustic propagation problems governed by Eq. (1). The computational subdomain $\Omega_{P S}$ is discretized in an equidistant mesh with a grid spacing $\Delta x_{P S}$ determined by the smallest wavelength of interest. The total subdomain dimension is $L_{t o t, P S}=\left(N_{P S}-1\right) \Delta x_{P S}$, with $N_{P S}$ the total number of grid points. The gradients of the acoustic variables of Eqs. (2) are calculated in the wavenumber domain using Eqs. (3), by multiplying the transformed discrete variables by the derivative operator $i k_{n}$, with $i$ the imaginary number and $k_{n}$ the wavenumber vector defined in Eq. (4). The transformation of the discrete acoustic variables is done by using Fourier analysis and synthesis, where $\mathcal{F}$ and $\mathcal{F}^{-1}$ are the forward and inverse discrete Fourier transform defined in Eqs. (5).

$$
\begin{gathered}
\left.\frac{\partial p}{\partial x}\right|_{x_{l}}=\mathcal{F}^{-1}\left(i k_{n} \mathcal{F}\left(p\left(x_{l}\right)\right)\right), 0 \leq l \leq N_{P S}-1, \\
\left.\frac{\partial u_{x}}{\partial x}\right|_{x_{l}}=\mathcal{F}^{-1}\left(i k_{n} \mathcal{F}\left(u_{x}\left(x_{l}\right)\right)\right), 0 \leq l \leq N_{P S}-1 . \\
k_{n}=\frac{2 \pi n}{N_{P S} \Delta x_{P S}}, n \in\left[-\frac{N_{P S}}{2}+1,-\frac{N_{P S}}{2}+2, \ldots, \frac{N_{P S}}{2}\right] .
\end{gathered}
$$




$$
\begin{aligned}
\mathcal{F}(\ldots) & =\Delta x_{P S} \sum_{l=0}^{N_{P S}-1}(\ldots) e^{-i k_{n} x_{l}}, \\
\mathcal{F}^{-1}(\ldots) & =\frac{1}{N_{P S} \Delta x_{P S}} \sum_{n=-N_{P S} / 2+1}^{N_{P S} / 2}(\ldots) e^{i k_{n} x_{l}} .
\end{aligned}
$$

The time derivatives of the governing equations are computed using the optimized six-stage low-storage Runge-Kutta scheme presented by Bogey and Bailly, ${ }^{20}$ referred to as RKo6s, and used in previous Fourier PSTD applications. ${ }^{2,8}$ The solutions are updated every time step $\Delta t_{P S}=\nu_{P S} \Delta x_{P S} / c_{0}$, where $\nu_{P S}$ is the Courant number.

\section{Nodal DG time-domain method}

Discontinuous Galerkin methods are based on local polynomial approximations of degree $N$. The computational subdomain $\Omega_{D G}$ is divided into a subset of $T$ non-overlapping conforming elements. The method approximates the variables by the direct sum of the local polynomials solutions in each element and uses the so-called numerical flux $\hat{\mathbf{f}}^{\partial T}$ to achieve a unique solution at the elements interface $\partial T$. Additionally, the numerical flux allows to prescribe boundary conditions where $\partial T$ is a boundary of $\Omega_{D G}$. For simplex elements in 1D, i.e. segments, the number of grid points in one element is $N_{p}=N+1$. In the current work, the DG solutions are computed using nodal Jacobi polynomials at the Legendre-Gauss-Lobatto quadrature points.

The semi-discrete formulation of the LEE (Eq. (1)), following the work by Hu et al., ${ }^{21}$ Toulorge et al. ${ }^{14}$ and Reymen et al. ${ }^{13}$ is shown in Eq. (6).

$$
\mathbf{M}^{T} \frac{\partial \mathbf{q}^{T}}{\partial t}-\sum_{j=1}^{d} \mathbf{K}_{j}^{T} \mathbf{A}_{j}^{T} \mathbf{q}^{T}+\sum_{i=1}^{b} \mathbf{M}^{\partial T_{i}} \hat{\mathbf{f}}^{\partial T_{i}}+\mathbf{M}^{T} \mathbf{C}^{T} \mathbf{q}^{T}=0,
$$

where $\mathbf{M}^{T}$ and $\mathbf{K}^{T}$ represent the mass and stiffness matrices to compute the spatial derivatives, respectively and $\mathbf{A}_{\mathbf{j}}{ }^{T}$ and $\mathbf{C}^{T}$ are defined in section II.A. Indexes $d$ and $b$ represent the dimensionality of the problem and the number of faces of the elements, respectively.

Various types of fluxes have been proposed and used in literature for aeroacoustics applications. Along this work, the commonly used numerical Lax-Friedrichs flux, as defined in Eq. (7), has been used. In Eq. (7), $\alpha_{\Lambda}$ represents the largest characteristic velocity of the LEE, obtained from matrix 8. When the fluxes in matrix $A_{j}$ are projected in a normal direction $\mathbf{n}$, the fluxes can be reduced to the diagonal matrix (8), containing the characteristics speeds as a function of the normal velocity of the mean flow and the speed of sound. Additionally, in Eq. (7), $T^{\prime}$ is the element sharing the interface $\partial T$ with $T$ and $\mathbf{f}^{T}=\mathbf{A}_{\mathbf{j}}{ }^{T} \mathbf{q}^{T}$.

$$
\begin{gathered}
\hat{\mathbf{f}}^{\partial T}=\frac{1}{2}\left[\left(\mathbf{f}^{T}+\mathbf{f}^{T^{\prime}}\right) \cdot \mathbf{n}-\alpha_{\Lambda}\left(\mathbf{q}^{T}-\mathbf{q}^{T^{\prime}}\right)\right] . \\
\boldsymbol{\Lambda}=\left[\begin{array}{ccccc}
u_{0, n} & 0 & 0 & 0 & 0 \\
0 & u_{0, n} & 0 & 0 & 0 \\
0 & 0 & u_{0, n} & 0 & 0 \\
0 & 0 & 0 & u_{0, n}+c_{0} & 0 \\
0 & 0 & 0 & 0 & u_{0, n}-c_{0}
\end{array}\right] .
\end{gathered}
$$

The numerical DG methodology is implemented following the quadrature-free approach introduced by Atkins and Shu ${ }^{16}$ and following the algorithms for the nodal approach presented by Hesthaven and Warburton. ${ }^{17}$ The optimized forth-order eight-stage time scheme (RKF84) derived by Toulorge and Desmet ${ }^{14}$ is implemented to compute the time derivatives of the governing equations. Due to the hybridization process (see section III), the time step $\Delta t_{D G}$ in DG will be the largest integer fraction of $\Delta t_{P S}$ that still obeys the stability condition. ${ }^{14}$ 


\section{Hybridization process}

The hybridization process is presented for the one-dimensional governing Eqs. (2) on a non-staggered or collocated grid. The one-dimensional physical domain $\Omega=\left\{x \in\left[x_{\text {left }}, x_{\text {right }}\right]\right\}$ is divided into two numerical subdomains with an overlapping area or copy zone, as shown in figure 3 , with $x_{l e f t, D G}=x_{l e f t}$ and $x_{\text {right, } P S}=x_{\text {right }}$ :

$$
\begin{array}{r}
\Omega_{P S}=\left\{x_{P S} \in\left[x_{\text {left }, P S}, x_{\text {right }, P S}\right]\right\}, \\
\Omega_{D G}=\left\{x_{D G} \in\left[x_{\text {left }, D G}, x_{\text {right }, D G}\right]\right\}, \\
\Omega_{c z}=\left\{x_{c z} \in\left[x_{\text {left }, P S}, x_{\text {right }, D G}\right]\right\} .
\end{array}
$$

The subdomains are discretized using the same element size, i.e. $\Delta x_{P S}=\Delta x_{D G}$. The size of the elements is determined by the smallest wavelength or maximum frequency of interest. The maximum frequency in Fourier PSTD $f_{\max , P S}$ is limited by the spatial Nyquist condition and is related to the grid spacing according to the following expression $f_{\max , P S}=c_{o} /\left(2 \Delta x_{P S}\right)$. This condition is known as the two points per wavelength condition $\left(N_{\lambda}=2\right)$. In this work, the results of the hybrid method are presented up to $f_{\max , h y b}=c_{0} /\left(2.5 \Delta x_{P S}\right)$, what is considered to be a reasonable range. The total number of elements in the numerical subdomains are $T_{P S}=N_{P S}-1$ and $T_{D G}$. The copy zone area has $T_{c z}$ elements and it is divided in two parts as shown in figure 4: one area $(c z P S D G)$ where the values of the Fourier PSTD solutions are copied to DG with $T_{c z P S D G}$ elements and a second zone $(c z D G P S)$ where the DG values are transferred to Fourier PSTD with $T_{c z D G P S}$ elements.

As part of the coupling algorithm, the field Fourier PSTD variables are multiplied by a Gaussian window to obtain spatial periodicity and minimize the wrap-around effects due to the Fourier kernels of the spatial derivative operator. The window is built using Eq. (9) and following the indications from Hornikx et al. ${ }^{8}$ The single sided exponential part of the window has $N_{w}$ number of points and is coincident with the coupling area from DG to Fourier PSTD $\left(T_{c z D G P S}=T_{w}=N_{w}-1\right)$.

$$
w\left(l_{w}, N_{w}\right)=e^{-\alpha_{w} \ln (10)\left(\frac{l_{w}-N_{w}}{N_{w}}\right)^{2 \beta_{w}}}, 0 \leq l_{w} \leq N_{w}-1 .
$$

No boundary conditions are imposed at $x_{l e f t, P S}$, nor at $x_{\text {right,DG }}$. Acoustically rigid boundary conditions are implemented at $x_{l e f t, D G}$. The conditions are weakly imposed in the numerical flux at the domain boundary by setting a zero velocity value normal to the boundary $\mathbf{u} \cdot \mathbf{n}=0$. The spectral interpolation of the Fourier PSTD solutions to obtain the values of the acoustic variables at the DG nodes is performed using Eqs. (10). The interpolation is done by a transformation of the Fourier PSTD solutions to the wavenumber domain, where the transformed variables are multiplied with the exponential that accounts for the location of the DG nodes. Since the grids of both solvers are conformal, the interpolation is only needed for the internal nodes of the DG elements and the distance from the Fourier PSTD nodes to each internal node is constant for every element. Therefore, for one-dimensional cases, $N-1$ interpolations of the Fourier PSTD solutions are needed. If $r_{p, j}$ contains the $j$ coordinates of the Legendre-Gauss-Lobatto nodes in the reference element, i.e. $r_{p, j} \in[-1,1]$, then the distances between the Fourier PSTD nodes to the DG internal nodes are calculated using Eq. (11).

$$
\begin{gathered}
p\left(x_{l}-\Delta x_{i n t, j}\right)=\mathcal{F}^{-1}\left(e^{i k_{n} \Delta x_{i n t, j}} \mathcal{F}\left(p\left(x_{l}\right)\right)\right), \\
u_{x}\left(x_{l}-\Delta x_{i n t, j}\right)=\mathcal{F}^{-1}\left(e^{i k_{n} \Delta x_{i n t, j}} \mathcal{F}\left(u_{x}\left(x_{l}\right)\right)\right) . \\
\Delta x_{i n t, j}=\left(r_{p, j}-1\right) \frac{\Delta x_{P S}}{2}, j=[2, \ldots, N p-1] .
\end{gathered}
$$

Since the hybrid approach couples the solutions over a zone rather than at the interface of the numerical methods, the solvers can use local time stepping without transferring data at intermediate steps. In this paper, following the work by Bogey and Bailly, ${ }^{20}$ the stability condition given by the Courant number $\nu_{P S}$ for the Fourier PSTD calculations is equal to 0.5 unless otherwise indicated. Whereas for DG, $\nu_{D G}$ is initially taken from the maximum Courant numbers given by Toulorge et al. ${ }^{14}$ for the Runge-Kutta RKF84 time scheme. These Courant numbers are more restrictive than $\nu_{P S}$ when the polynomial order is $N>2$. Therefore, the data are post-processed and exchanged after every Fourier PSTD time step $\Delta t_{P S}$. The final DG time step $\Delta t_{D G}$ is calculated after computing the minimum integer number $s_{\text {hyb }}$ of DG time steps in one 


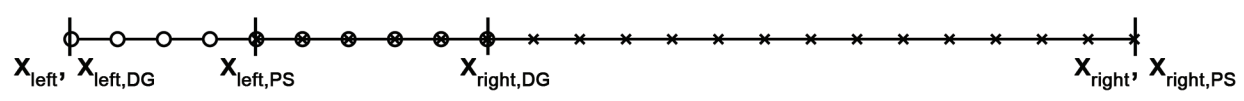

Figure 3: Sketch of a one-dimensional example of the hybrid mesh. The circles represent DG and the crosses Fourier PSTD (for clarity, the internal nodes of the DG elements are not represented in the figure).

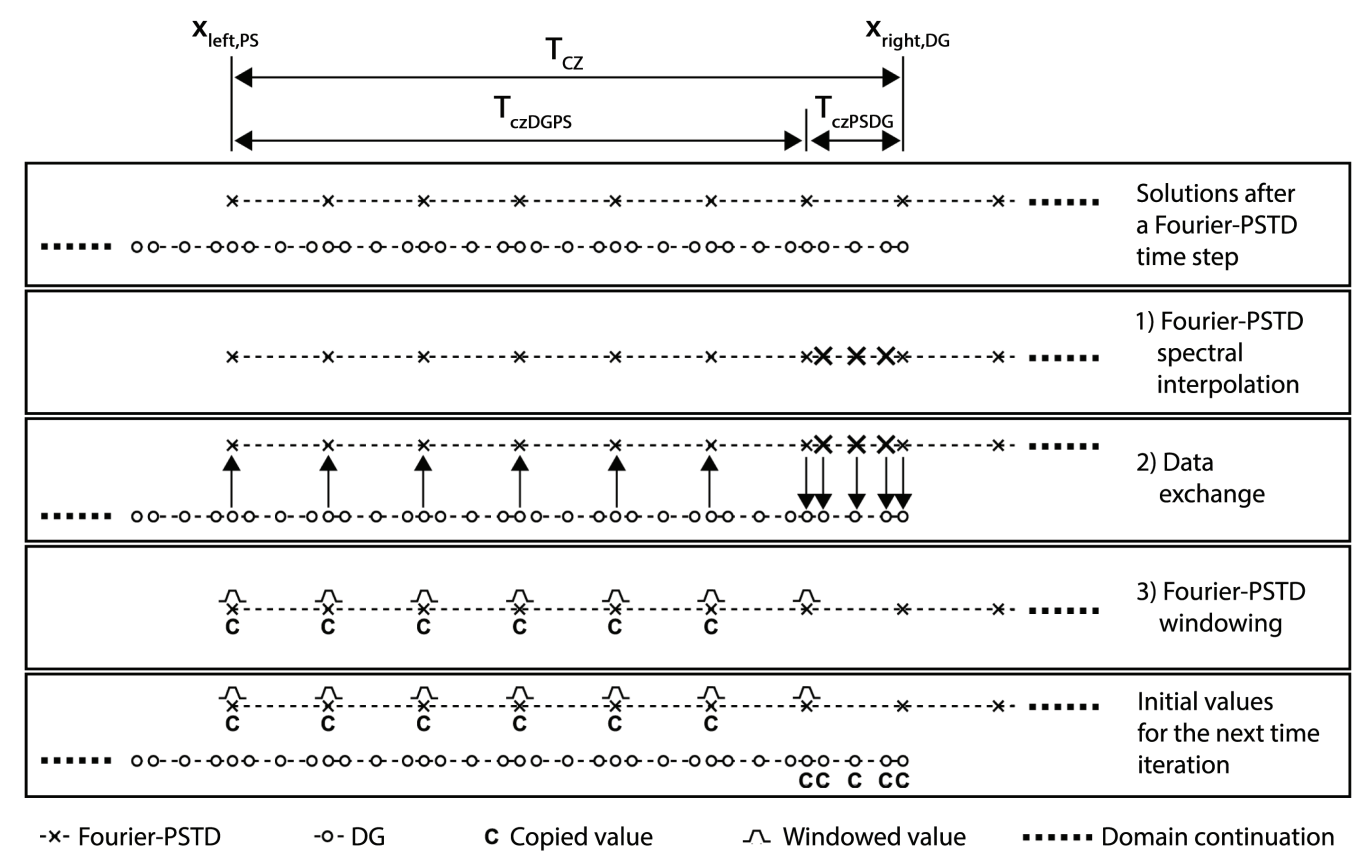

Figure 4: Post-processing and data exchange in the coupling zone of the hybrid method.

Fourier PSTD time step, such that the stability condition ${ }^{14}$ is fulfilled. In this work, $s_{h y b}$ is referred to as time step factor. This time process is schematically shown in Figure 5. The hybridization approach follows three main steps indicated in figure 4: Step 1) consists of performing spectral-interpolation of the Fourier PSTD solutions in order to find the values at the DG internal nodes in the $c z P S D G$ area. In step 2), data are exchanged between solvers in the data-exchange areas. Finally, the acoustic Fourier PSTD variables are multiplied by a Gaussian window in step 3) before computing the next time iteration.

\section{Error analysis}

To achieve a successful implementation of the hybrid methodology described in section III, it is needed to determine and quantify the main sources of error in order to select a suitable combination of parameters. In this section, the errors are discussed for the 1D hybrid methodology. For each error investigated in the subsequent sections, the other sources are kept constant but are still present in the evaluations.

The initial conditions, imposed in Fourier PSTD, are the broadband pressure and velocity distribution $p\left(x, t_{0}\right)=e^{-b_{s}\left(x-x_{s}\right)^{2}}, u\left(x, t_{0}\right)=-p\left(x, t_{0}\right) / \rho_{0} c_{0}$, where $b_{s}$ determines the bandwidth of the spectrum and $x_{s}$ the source location. In DG, the time-domain computation is initialized with a zero-valued pressure and velocity distribution. No special boundary conditions are imposed in the left side of the Fourier PSTD domain since the Gaussian window is used to enforce periodicity, whereas the right side is terminated using a perfectly match layer (PML) as in the paper by Hornikx et al. ${ }^{2}$ to avoid wrap-around effects and have a reflection free termination. To minimize the error contribution from the PML, a 100-elements thick layer has been used. On the other hand, the left side of the DG domain is computed using an acoustically hard boundary whereas the right end is where the values from Fourier PSTD are copied and no boundary 


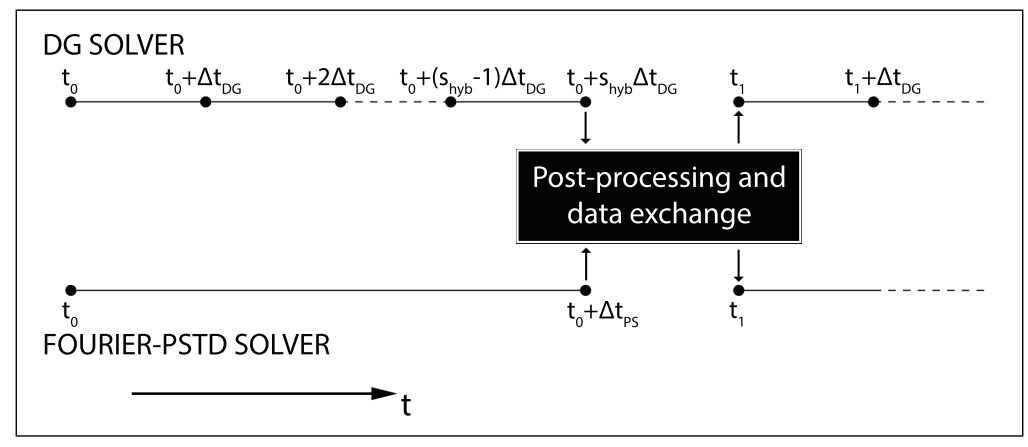

Figure 5: Time diagram of the hybridization process.

Table 1: Main parameters of the hybrid model used for the error evaluation

\begin{tabular}{l|c} 
Element size $\left(\Delta x_{P S}=\Delta x_{D G}\right)$ & $0.0343 \mathrm{~m}$ \\
Hybrid domain dimensions & $612 \Delta x_{P S} \mathrm{~m}$ \\
Number of DG elements $\left(T_{D G}\right)$ & 103 elements \\
Excitation-recording distance & $321 \Delta x_{P S} \mathrm{~m}$ \\
Excitation-recording distance reference $\left(x_{s, r e f}\right)$ & $29 \Delta x_{P S} \mathrm{~m}$ \\
Source parameter $\left(b_{s}=b_{s, r e f}\right)$ & $80 \mathrm{~m}^{-2}$
\end{tabular}

conditions are imposed at that end. The reflected wave from DG is then recorded at a position $x_{r}$ in the Fourier PSTD domain during a certain recording time $\left(t_{r e c}\right)$. The errors are computed by transforming the recorded acoustic variables to the frequency domain $\left(Q\left(f_{i}\right)\right)$ using a forward Fourier transform. The amplitude and phase or dispersion errors are calculated from the acoustic variables in the frequency domain using Eqs. (12) and (13), respectively.

$$
\begin{gathered}
\epsilon_{\text {amp }}\left(f_{i}\right)=20 \log _{10}\left|\frac{\left|Q_{\text {ref }}\left(f_{i}\right)\right|-\left|Q_{\text {calc }}\left(f_{i}\right)\right|}{\left|Q_{\text {ref }}\left(f_{i}\right)\right|}\right| ; f_{i} \in\left[f_{1}, f_{2}\right], \\
\epsilon_{\phi}\left(f_{i}\right)=20 \log _{10}\left(\frac{\left|\phi\left[Q_{\text {ana }}\left(f_{i}\right)\right]-\phi\left[Q_{\text {calc }}\left(f_{i}\right)\right]\right|}{\pi}\right) ; f_{i} \in\left[f_{1}, f_{2}\right],
\end{gathered}
$$

where, $Q_{r e f}$ is the numerical solution at a reference receiver close to the source, $Q_{a n a}$ is the analytical solution and $Q_{\text {calc }}$ is the solution from the hybrid method. In this work, $Q_{r e f}$ is used in the evaluation of the amplitude error instead of $Q_{a n a}$, to exclude the initial conditions imposed in Fourier PSTD as a possible additional source of error. All the errors are computed from the sound pressure solutions $\left(Q\left(f_{i}\right)=P\left(f_{i}\right)\right)$ and, unless otherwise indicated, the main parameters of the hybrid model used for the error evaluation are presented in table 1. In the current work, the number of elements in the DG subdomain $\left(T_{D G}\right)$ is fixed for all calculations whereas the number of elements in Fourier PSTD $\left(T_{P S}\right)$ changes as a function of the size of the copy zones. In this way, the travelling time of the wave in the DG subdomain is kept constant and thereby the error contribution from the DG solver for a fixed polynomial order.

\section{A. The error related to the coupling: DG to Fourier PSTD}

As mentioned in previous sections, the hybrid methodology uses a Gaussian window to keep spatial periodicity in the Fourier PSTD domain after transferring values from DG. In this work, the error contribution of this coupling includes both actions, copying values and windowing. The influence of the main parameters of the data-exchange area $\left(T_{c z D G P S}\right)$ and the Gaussian window $\left(T_{w}, \alpha_{w}\right.$ and $\left.\beta_{w}\right)$ are investigated in this paper. Along this section, the reference to the size of the data-exchange area $T_{c z D G P S}$ is omitted, using instead the reference to the window size $T_{w}$, since $T_{c z D G P S}=T_{w}$ in this work. In order to minimize the errors arising

from the temporal scheme, a small time step is used in these calculations. The main parameters of this hybrid model are shown in table 2. The amplitude and dispersion coupling errors are computed using Eqs. 
Table 2: Main parameters of the hybrid model used for the error evaluation of the coupling from DG to Fourier PSTD

\begin{tabular}{l|c} 
Fourier PSTD Courant number $\left(\nu_{P S}\right)$ & 0.05 \\
Time step factor $\left(s_{h y b}\right)$ & 1 \\
Recording time $\left(t_{r e c}\right)$ & $19260 \Delta t_{P S} \mathrm{~s}$ \\
DG polynomial order $(N)$ & 10 \\
Copy zone czPSDG size $\left(T_{c z P S D G}\right)$ & 1 element \\
Gaussian window size $\left(T_{w}=T_{c z D G P S}\right)$ & {$[10: 1: 100]$ elements } \\
Gaussian window parameter $\left(\alpha_{w}\right)$ & {$[1: 1: 10]$} \\
Gaussian window parameter $\left(\beta_{w}\right)$ & {$[2: 1: 10]$}
\end{tabular}

(14) and (15), respectively. In the model used for this error evaluation, the wave travels twice through the Gaussian window: on the way to the boundary and back to the Fourier PSTD domain after being reflected. Hence, the errors are normalized to twice the Gaussian window length $\left(2 T_{w}\right)$.

$$
\begin{gathered}
\epsilon_{w, a m p}\left(f_{1}, f_{2}\right)=10 \log _{10}\left(\frac{10^{\sup \left(\epsilon_{a m p}\left(f_{i}\right) / 10\right)}}{2 T_{w}}\right), f_{i} \in\left[f_{1}, f_{2}\right] . \\
\epsilon_{w, \phi}\left(f_{1}, f_{2}\right)=10 \log _{10}\left(\frac{10^{\sup \left(\epsilon_{\phi}\left(f_{i}\right) / 10\right)}}{2 T_{w}}\right), f_{i} \in\left[f_{1}, f_{2}\right] .
\end{gathered}
$$

In Appendix A, figures 9 and 10 present the results of $\epsilon_{w, a m p}\left(f_{1}, f_{2}\right)$ and $\epsilon_{w, \phi}\left(f_{1}, f_{2}\right)$ computed in the full frequency range of interest $\left(0, f_{s} / 2.5\right)$ (columns of graphs (a) and (b)) and for a low frequency range $\left(0, f_{s} / 64\right)$ (columns of graphs (c) and (d)). The errors are plotted for different values of $T_{w}, \alpha_{w}$ and $\beta_{w}$. In general, the results in the full frequency range exhibit a linear behaviour between the errors and the window size until a certain error bound where the results seem to converge for most cases. Therefore, linear regressions of the data points are calculated and included in the columns (a) and (b) of the figures. The regression analysis has been computed using the range of data points from $T_{w, l o w}=10$ elements to the upper limits $T_{w, u p}$ given in tables 7 and 10 for the amplitude and phase errors, respectively. The values of the slope $\left(b_{w}\right)$ and intercept $\left(a_{w}\right)$ of the regression lines for the different combinations of $\alpha_{w}$ and $\beta_{w}$ are presented in tables 8 and 9 for the amplitude error, and, 11 and 12 for the dispersion error. The values correspond to Eqs. (16) and (17), respectively.

$$
\begin{gathered}
\epsilon_{w, a m p}\left(0, f_{s} / 2.5\right)=a_{w, a m p}+b_{w, a m p} T_{w} \\
\epsilon_{w, \phi}\left(0, f_{s} / 2.5\right)=a_{w, \phi}+b_{w, \phi} T_{w} .
\end{gathered}
$$

The intercepts of the linear regressions show that for a fixed value of $\alpha_{w}$, higher values of $\beta_{w}$ give a better performance of the hybrid method (see tables 9 and 12). However, the slopes in tables 8 and 11 show that, when $\beta_{w}$ is increased, a bigger window size $T_{w}$ is needed to achieve the same amount of reduction. Furthermore, the linear regressions are only valid to a certain lower error bound, around $-75 d B$ for most combinations of $\alpha_{w}$ and $\beta_{w}$ of the dispersion error and more difficult to determine as a single value for the amplitude error. On the other hand, when a fixed value of $\beta_{w}$ is selected from tables 9 and 12 , the differences among the error results for the different $\alpha_{w}$ values are almost negligible for most cases.

Regarding the results in the low frequency range $\left(0, f_{s} / 64\right)$ shown in columns of graphs (c) and (d) of figures 9 and 10, the amplitude and dispersion errors have a lower bound depending on the window parameters. The floor of the errors is bounded around $-220 d B$ and $-165 d B$ for amplitude and dispersion errors, respectively. These bounds are only reached for high values of $\alpha_{w}$. Clearly, lower values of $\beta_{w}$ (except for values 2 and 3, which have a different tendency) need less window elements to reach the bounds. In any case, since the errors in this frequency range are already very low, the analysis is of less relevance.

According to the analysis done in this section, the Gaussian window parameters $\alpha_{w}=10$ and $\beta_{w}=4$ seem to be a suitable combination for the computed model and will be used in the following calculations. 


\section{B. The error related to the coupling: Fourier PSTD to DG}

The influence of the size of the data-exchange area from Fourier PSTD to DG in the errors $\epsilon_{w, a m p}\left(0, f_{s} / 2.5\right)$ and $\epsilon_{w, \phi}\left(0, f_{s} / 2.5\right)$ is investigated in this section. The methodology is in line with the one presented in the previous section but, in this case, different values of the size of the copy zone from Fourier PSTD to DG $T_{c z P S D G}=[1: 1: 8]$ are investigated for the following Gaussian window parameters $T_{w}=[10: 10: 90]$, $\alpha_{w}=10$ and $\beta_{w}=4$. The rest of the parameters used in this model are the ones presented in tables 1 and 2. The errors are computed using Eqs. (14) and (15). In general, no significant variations are found in the results presented in tables 3 and 4 when the number of elements of the copy zone $T_{c z P S D G}$ is increased. These results are consistent for the different calculated cases of $T_{w}$.

Table 3: Amplitude error $\epsilon_{w, a m p}\left(0, f_{s} / 2.5\right)[d B]$ for different sizes of the copy zone from Fourier PSTD to DG $\left(T_{c z P S D G}\right)$. The error is presented for different values of the size of the Gaussian window $\left(T_{w}\right)$

\begin{tabular}{c|cccccccc} 
& \multicolumn{7}{|c}{$T_{c z P S D G}$} \\
$T_{w}$ & 1 & 2 & 3 & 4 & 5 & 6 & 7 & 8 \\
\hline 10 & -24.3 & -24.3 & -24.3 & -24.2 & -24.3 & -24.2 & -24.3 & -24.2 \\
20 & -30.6 & -30.6 & -30.6 & -30.7 & -30.8 & -30.9 & -31.1 & -31.0 \\
30 & -45.9 & -45.9 & -45.8 & -45.6 & -45.4 & -45.8 & -45.7 & -46.0 \\
40 & -52.6 & -52.4 & -52.2 & -52.3 & -52.9 & -52.6 & -52.9 & -53.0 \\
50 & -66.4 & -66.7 & -66.8 & -66.9 & -68.6 & -66.6 & -66.5 & -66.4 \\
60 & -72.7 & -72.8 & -73.0 & -73.2 & -70.8 & -73.8 & -73.8 & -73.1 \\
70 & -85.6 & -85.8 & -86.0 & -86.4 & -78.3 & -88.7 & -84.2 & -86.5 \\
80 & -97.5 & -97.8 & -97.8 & -97.9 & -81.3 & -92.9 & -90.2 & -94.5 \\
90 & -103.9 & -104.2 & -104.5 & -104.8 & -82.6 & -97.1 & -90.7 & -95.0
\end{tabular}

Table 4: Dispersion error $\epsilon_{w, \phi}\left(0, f_{s} / 2.5\right)[d B]$ for different sizes of the copy zone from Fourier PSTD to DG $\left(T_{c z P S D G}\right)$. The error is presented for different values of the Gaussian window size $\left(T_{w}\right)$

\begin{tabular}{c|cccccccc} 
& \multicolumn{7}{|c}{$T_{c z P S D G}$} \\
$T_{w}$ & 1 & 2 & 3 & 4 & 5 & 6 & 7 & 8 \\
\hline 10 & -31.4 & -31.5 & -31.5 & -31.5 & -31.5 & -31.4 & -31.5 & -31.4 \\
20 & -46.4 & -46.4 & -46.6 & -46.4 & -46.4 & -46.3 & -46.4 & -46.4 \\
30 & -51.8 & -52.0 & -52.1 & -52.0 & -51.9 & -51.8 & -52.1 & -51.9 \\
40 & -64.8 & -64.9 & -64.7 & -64.6 & -65.1 & -64.7 & -65.2 & -64.7 \\
50 & -74.4 & -74.1 & -74.0 & -74.0 & -75.1 & -73.9 & -75.0 & -74.0 \\
60 & -78.7 & -78.4 & -78.3 & -78.4 & -80.5 & -78.5 & -80.1 & -78.3 \\
70 & -79.1 & -78.9 & -78.7 & -78.5 & -80.3 & -78.3 & -80.2 & -78.2 \\
80 & -81.0 & -80.9 & -80.7 & -80.6 & -82.6 & -80.2 & -82.0 & -79.9 \\
90 & -81.9 & -81.8 & -81.6 & -81.5 & -83.4 & -81.1 & -82.9 & -80.8
\end{tabular}

\section{Error of the hybrid method compared with the Fourier PSTD standalone solver}

In order to obtain the precision of the hybrid approach, the error results computed in this section are compared with the results of an equivalent model calculated with a Fourier PSTD standalone solver. The wave is travelling the same distance as in the hybrid model but in free field for the standalone solver, i.e. no reflections from the boundaries. The rest of the features of the models are equivalent and the main parameters are presented in tables 1 and 5, where the Gaussian window parameters $\alpha_{w}=10$ and $\beta_{w}=4$ have been selected after the analysis done in section IV.A as a suitable combination for the computed model. The dissipation and dispersion error of both methods, hybrid $\left(\epsilon_{a m p, h y b}\right.$ and $\left.\epsilon_{\phi, h y b}\right)$ and standalone Fourier PSTD $\left(\epsilon_{a m p, P S}\right.$ and $\left.\epsilon_{\phi, P S}\right)$, are computed using Eqs. (12) and (13), respectively. The total errors of the 
Table 5: Main parameters of the hybrid and Fourier PSTD standalone models used for the total error evaluation of the hybrid methodology

Fourier PSTD Courant number $\left(\nu_{P S}\right)$
Recording time $\left(t_{r e c}\right)$
DG polynomial order $(N)$
Copy zone czPSDG size $\left(T_{c z P S D G}\right)$
Gaussian window size $\left(T_{w}=T_{c z D G P S}\right)$
Gaussian window parameter $\left(\alpha_{w}\right)$
Gaussian window parameter $\left(\beta_{w}\right)$

0.5

$1926 \Delta t_{P S} \mathrm{~S}$

$[2: 1: 10]$

1 element

[10:1:100] elements

10

4

hybrid methodology, referred to the standalone solver results, are calculated using Eqs. (18) and (19). The results are computed in the full frequency range of interest $\left(0, f_{s} / 2.5\right)$ and for a low frequency range $\left(0, f_{s} / 64\right)$. The performance of the hybrid method is investigated using different polynomial orders $(N)$ in the DG solver. The time step factors $s_{h y b}$ corresponding to each polynomial order computation are shown in table 6 .

$$
\begin{gathered}
\epsilon_{t o t, a m p}\left(f_{1}, f_{2}\right)=10 \log _{10}\left(\frac{10^{\sup \left(\epsilon_{\text {amp }, h y b}\left(f_{i}\right) / 10\right)}}{10^{\sup \left(\epsilon_{\text {amp }, P S}\left(f_{i}\right) / 10\right)}}\right), f_{i} \in\left[f_{1}, f_{2}\right] . \\
\epsilon_{t o t, \phi}\left(f_{1}, f_{2}\right)=10 \log _{10}\left(\frac{10^{\sup \left(\epsilon_{\phi, h y b}\left(f_{i}\right) / 10\right)}}{10^{\sup \left(\epsilon_{\phi, P S}\left(f_{i}\right) / 10\right)}}\right), f_{i} \in\left[f_{1}, f_{2}\right] .
\end{gathered}
$$

The first results obtained with the hybrid methodology in this section have shown the necessity of filtering the Fourier PSTD solutions in order to minimize numerical instabilities arising from the Fourier PSTD method, similar as reported by Hornikx et al. ${ }^{3}$ A low-pass Gaussian frequency filter is therefore used to filter out the high frequency components of the Fourier PSTD solutions after every time step. The cut-off frequency of the Gaussian filter is set to $f_{\text {filt }}=c_{0} / 2.1 \Delta x_{P S}$, with $\alpha_{\text {filt }}=4$ and $\beta_{\text {filt }}=4$. The filter is equivalent to the one used by Hornikx et al. ${ }^{3}$ for one-dimensional cases.

The results are presented in figures 6 and 7 . In these figures, the calculations for $N=2$ and $N=3$ are not included since the computed errors are too high to be of interest. For the full frequency range (figure 6 ), it is clear that polynomial orders $N \geq 5$ have a very similar performance and the amplitude error (figure figure 6 a)) is below the standalone computation for window sizes $T_{w} \geq 30$ elements. Regarding the phase error in this frequency range (figure $6 \mathrm{~b}$ )), the different polynomial orders have almost no influence in the results of the hybrid methodology and the hybrid errors are below the standalone solver errors for all window sizes. The amplitude error in the low frequency range (figure 7 a)) show that the hybrid method performs better for lower N. In this case, a bigger window size is needed in the hybrid method to keep the errors below the errors of standalone solver. Regarding the phase error, shown in figure figure $7 \mathrm{~b}$ ), it keeps the same tendency as the former analysis for the full frequency range, except for window sizes $T_{w} \leq 12$ elements where the errors are bigger than those of the standalone solver. In any case, the errors in the low frequency range are already very low and, therefore, of less relevance.

Overall, polynomial order 5 seems to be a suitable selection and is used in the following analysis together with a window size of $T_{w}=T_{c z D G P S}=30$ elements that appears to be the smallest size where the maximum error over the whole frequency range is still below the error of the standalone solver. These results are presented in figure 8 for $\epsilon_{a m p, h y b}$ and $\epsilon_{\phi, h y b}$ together with the results of the standalone solver, $\epsilon_{a m p, P S}$ and $\epsilon_{\phi, P S}$, in the frequency range of interest (indicated as points per wavelength $N_{\lambda}$ in this figure). Additionally, figure 8 includes the Runge-Kutta analytical error of the Fourier PSTD standalone solver. The analytical RK error has been computed following the indications of Bogey and Bailly. ${ }^{20}$ The figure shows that the error of the standalone solver is mainly caused by the RKo6s time scheme. The hybrid methodology presents no significant additional error when compared with the standalone Fourier PSTD solver. 
Table 6: Time step factors $s_{\text {hyb }}$ for the different polynomial order $N$ used in the computation of the total error of the hybrid methodology. The time step factors represent the number of DG time steps in one Fourier PSTD time step.

\begin{tabular}{l|ccccccccc} 
Polynomial order $(N)$ & 2 & 3 & 4 & 5 & 6 & 7 & 8 & 9 & 10 \\
\hline Time step factor $\left(s_{\text {hyb }}\right)$ & 1 & 2 & 2 & 3 & 4 & 5 & 7 & 8 & 9
\end{tabular}
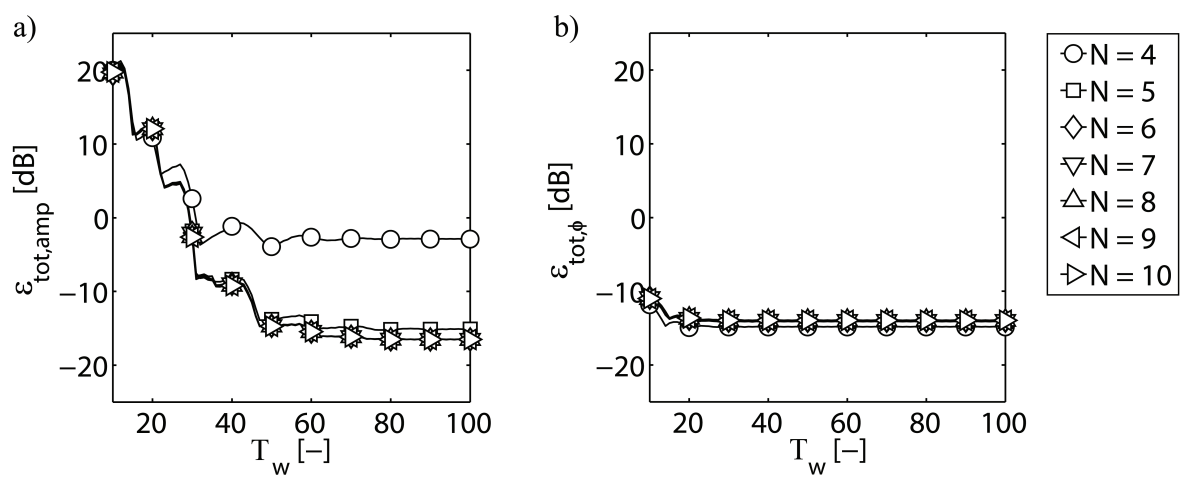

Figure 6: Total errors of the hybrid method in the full frequency range of interest referred to the errors of the standalone solver as a function of the number of elements of the Gaussian window $\left(T_{w}\right)$ for different polynomial order $(N)$. The figure includes a) the amplitude error $\epsilon_{t o t, a m p}\left(0, f_{s} / 2.5\right)$ and b) the phase error $\epsilon_{t o t, \phi}\left(0, f_{s} / 2.5\right)$.
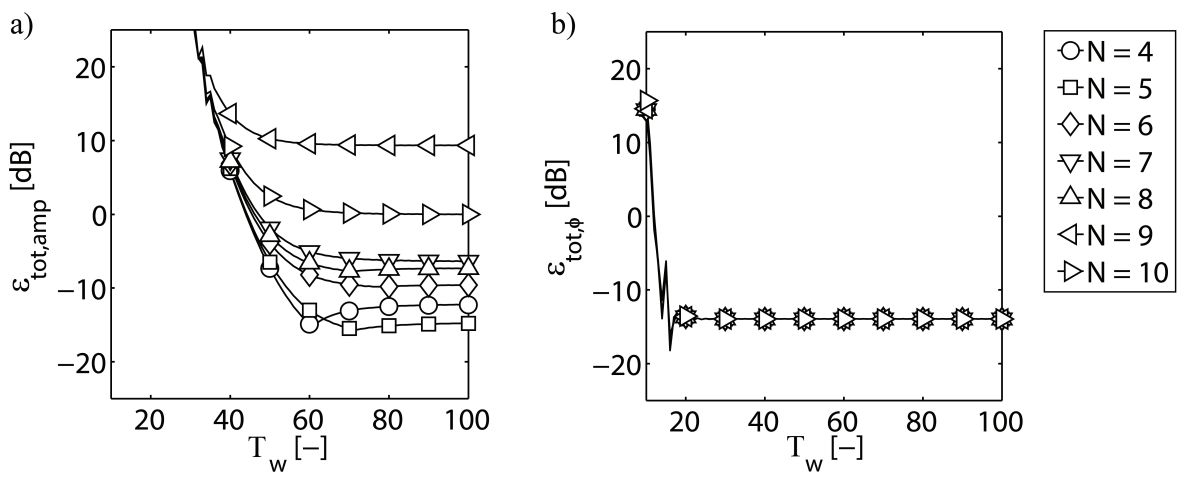

Figure 7: Total errors of the hybrid method in a low frequency range referred to the errors of the standalone solver as a function of the number of elements of the Gaussian window $\left(T_{w}\right)$ for different polynomial order $(N)$. The figure shows a) the amplitude error $\epsilon_{t o t, a m p}\left(0, f_{s} / 64\right)$ and b) the phase error $\epsilon_{\text {tot }, \phi}\left(0, f_{s} / 64\right)$.

\section{Conclusions}

A novel numerical hybrid approach is presented to solve the linearized Euler equations (LEE), coupling Fourier pseudospectral time-domain methodology (Fourier PSTD) with the nodal discontinuous Galerkin (DG) method. The scope of the hybrid approach is to allow the computation of arbitrary boundary conditions and complex geometries by using the benefits of the DG methodology at the boundaries while keeping Fourier PSTD in the bulk of the domain. The approach is presented for a one-dimensional case where the computational domain is decomposed into two subdomains with an overlapping area. The solutions of the LEE are approximated in each subdomain by one of the numerical methods and the hybrid method couples the results of both solvers in the overlapping area. The approach is based on conformal meshes to avoid spatial interpolation of the DG solutions, however an spatial interpolation scheme is needed in the 

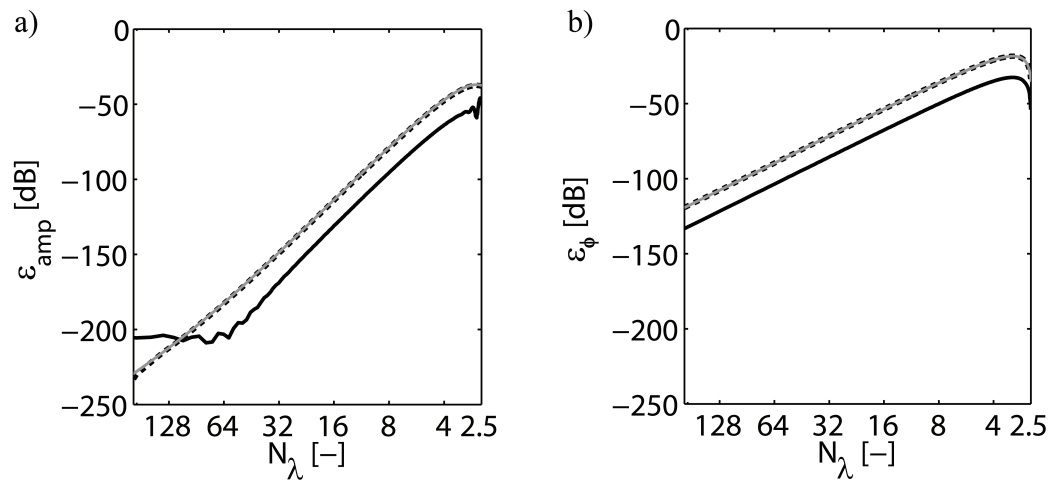

- Hybrid method $\cdots$ Fourier-PSTD standalone - Analytical RK error (RKo6s)

Figure 8: Total error of the hybrid method as function of points per wavelength $\left(N_{\lambda}\right)$ for DG order of polynomial $N=5$ and a window size of $T_{w}=T_{c z D G P S}=30$ elements. The figure includes the error of the standalone Fourier PSTD solver and the analytical Runge-Kutta (RKo6s) error. The figure shows a) the amplitude error $\epsilon_{a m p, h y b}\left(c_{0} /\left(N_{\lambda, i} \Delta x_{P S}\right)\right), N_{\lambda, i} \in[2.5,200]$ and b) the phase error $\epsilon_{\phi, h y b}\left(c_{0} /\left(N_{\lambda, i} \Delta x_{P S}\right)\right), N_{\lambda, i} \in[2.5,200]$.

hybridization process for the Fourier PSTD solutions in order to obtain the values at the internal nodes of the DG elements. The coupling algorithm includes the multiplication of the Fourier PSTD solutions by a Gaussian window function to impose periodicity after transferring the data from DG. Hence, the hybrid approach couples the solutions over a zone (overlapping area) rather than at the interface of the numerical methods. Therefore, the solvers can use local time stepping without transferring data at intermediate steps. Since the stability condition of DG is more restrictive than in Fourier PSTD, the data update between the solvers is only done after the larger (Fourier PSTD) time steps. Overall, the novel hybrid methodology shows no significant additional error when compare with a Fourier PSTD standalone solver for a suitable selection of the main parameters. The global error of the hybrid method is mainly dominated by the Runge-Kutta time scheme of the Fourier PSTD solver when the DG polynomial order is $N \geq 5$ and the number of elements of the copy zone from DG to Fourier PSTD and, consequently, of the Gaussian window is high enough. In the low frequency range, the amplitude error is influenced by the DG polynomial order but the errors in this frequency range are already very low and, therefore, of less relevance. In order to investigate the error contribution from the copy zones and the window function, the Runge-Kutta error has been reduced by selecting a low Fourier PSTD time step. In this way, the error sensitivity to the main parameters of the coupling areas has been investigated. The results of this analysis are presented to facilitate a suitable selection of the features of the data-transfer areas. In general, no significant variations are found in the errors when the size of the copy zone from Fourier PSTD to DG is investigated. In contrast, the size of the data-transfer area from DG to Fourier PSTD and the Gaussian window parameters are of main influence in the error results, especially if not enough elements are used. Additionally, linear regressions of the error data points are included to facilitate the understanding and usage of some of the results. Further developments of the hybrid methodology are in progress, mainly related with the implementation of the method for higher dimensions, the hybridization of the staggered case and the stability analysis.

\section{Appendix}

\section{A. Coupling error results: DG to Fourier PSTD}


Table 7: Upper limits of the number of elements $T_{w, u p}$ used to calculate the linear regression of $\epsilon_{w, a m p}\left(0, f_{s} / 2.5\right)$ data points $\left(T_{w, l o w}=10\right.$ elements for all cases $)$

\begin{tabular}{c|ccc} 
& \multicolumn{3}{|c}{$\alpha_{w}$} \\
$\beta_{w}$ & 1 & 2 & $>3$ \\
\hline 2 & 12 & 28 & 29 \\
3 & 21 & 36 & 50 \\
4 & 31 & 49 & 60 \\
5 & 39 & 59 & 60 \\
6 & 45 & 69 & 100 \\
7 & 54 & 79 & 100 \\
8 & 64 & 89 & 100 \\
9 & 69 & 100 & 100 \\
10 & 74 & 100 & 100
\end{tabular}

Table 8: Slope $b_{w, a m p}$ (see Eq. (9)) of the linear regression of $\epsilon_{w, a m p}\left(0, f_{s} / 2.5\right)$ data points

\begin{tabular}{c|cccccccccc} 
& \multicolumn{10}{|c}{$\alpha_{w}$} \\
$\beta_{w}$ & 1 & 2 & 3 & 4 & 5 & 6 & 7 & 8 & 9 & 10 \\
\hline 2 & -2.49 & -2.29 & -2.18 & -2.16 & -2.08 & -1.91 & -1.83 & -1.81 & -1.80 & -1.79 \\
3 & -1.78 & -1.75 & -1.60 & -1.54 & -1.47 & -1.41 & -1.36 & -1.34 & -1.32 & -1.30 \\
4 & -1.32 & -1.34 & -1.22 & -1.18 & -1.15 & -1.12 & -1.10 & -1.07 & -1.06 & -1.05 \\
5 & -1.04 & -1.07 & -1.00 & -0.98 & -0.95 & -0.93 & -0.92 & -0.91 & -0.90 & -0.89 \\
6 & -0.88 & -0.89 & -0.82 & -0.81 & -0.80 & -0.79 & -0.77 & -0.77 & -0.76 & -0.76 \\
7 & -0.75 & -0.77 & -0.72 & -0.71 & -0.70 & -0.69 & -0.68 & -0.68 & -0.67 & -0.67 \\
8 & -0.66 & -0.68 & -0.64 & -0.63 & -0.63 & -0.62 & -0.61 & -0.61 & -0.61 & -0.60 \\
9 & -0.60 & -0.61 & -0.58 & -0.58 & -0.57 & -0.56 & -0.56 & -0.56 & -0.55 & -0.55 \\
10 & -0.55 & -0.55 & -0.54 & -0.53 & -0.53 & -0.52 & -0.52 & -0.51 & -0.51 & -0.51
\end{tabular}

Table 9: Intercept $a_{w, a m p}$ (see Eq. (9)) of the linear regression of $\epsilon_{w, a m p}\left(0, f_{s} / 2.5\right)$ data points

\begin{tabular}{c|cccccccccc} 
& \multicolumn{1}{|c}{$\alpha_{w}$} \\
$\beta_{w}$ & 1 & 2 & 3 & 4 & 5 & 6 & 7 & 8 & 9 & 10 \\
\hline 2 & -6.3 & -8.1 & -6.5 & -4.3 & -3.6 & -4.8 & -5.0 & -4.4 & -3.7 & -3.1 \\
3 & -9.4 & -7.5 & -8.3 & -8.3 & -8.8 & -9.5 & -9.9 & -9.7 & -9.7 & -9.7 \\
4 & -11.7 & -10.0 & -11.2 & -11.3 & -11.4 & -11.5 & -11.6 & -11.9 & -11.9 & -12.0 \\
5 & -13.9 & -12.3 & -13.0 & -13.0 & -13.2 & -13.3 & -13.2 & -13.2 & -13.1 & -13.4 \\
6 & -15.0 & -14.0 & -15.4 & -15.0 & -15.0 & -15.0 & -15.2 & -15.2 & -15.2 & -15.2 \\
7 & -16.1 & -15.3 & -16.2 & -16.2 & -16.1 & -16.2 & -16.2 & -16.2 & -16.2 & -16.2 \\
8 & -17.2 & -16.4 & -17.0 & -16.9 & -17.0 & -17.0 & -17.0 & -17.0 & -16.9 & -17.0 \\
9 & -17.7 & -17.2 & -17.6 & -17.6 & -17.6 & -17.6 & -17.6 & -17.6 & -17.6 & -17.6 \\
10 & -18.2 & -17.9 & -18.2 & -18.1 & -18.1 & -18.1 & -18.1 & -18.1 & -18.2 & -18.1
\end{tabular}

14 of 18 
Table 10: Upper limits of the number of elements $T_{w, u p}$ used to calculate the linear regression of $\epsilon_{w, \phi}\left(0, f_{s} / 2.5\right)$ data points $\left(T_{w, l o w}=10\right.$ elements for all cases $)$

\begin{tabular}{c|cccccccccc} 
& \multicolumn{10}{|c}{$\alpha_{w}$} \\
$\beta_{w}$ & 1 & 2 & 3 & 4 & 5 & 6 & 7 & 8 & 9 & 10 \\
\hline 2 & 14 & 20 & 22 & 24 & 25 & 27 & 28 & 29 & 30 & 31 \\
3 & 23 & 28 & 30 & 32 & 33 & 34 & 35 & 36 & 37 & 37 \\
4 & 32 & 36 & 38 & 39 & 40 & 41 & 41 & 42 & 42 & 42 \\
5 & 33 & 36 & 38 & 39 & 40 & 41 & 41 & 42 & 42 & 42 \\
6 & 43 & 45 & 47 & 48 & 49 & 49 & 50 & 50 & 51 & 51 \\
7 & 43 & 45 & 47 & 48 & 49 & 49 & 50 & 50 & 51 & 51 \\
8 & 43 & 45 & 56 & 57 & 57 & 58 & 58 & 58 & 58 & 58 \\
9 & 43 & 45 & 56 & 57 & 57 & 58 & 58 & 58 & 58 & 58 \\
10 & 43 & 45 & 56 & 57 & 57 & 58 & 58 & 58 & 58 & 58
\end{tabular}

Table 11: Slope $b_{w, \phi}$ (see Eq. (9)) of the linear regression of $\epsilon_{w, \phi}\left(0, f_{s} / 2.5\right)$ data points

\begin{tabular}{c|cccccccccc} 
& \multicolumn{1}{|c}{$\alpha_{w}$} \\
$\beta_{w}$ & 1 & 2 & 3 & 4 & 5 & 6 & 7 & 8 & 9 & 10 \\
\hline 2 & -1.86 & -2.51 & -2.12 & -1.93 & -1.67 & -1.66 & -1.52 & -1.45 & -1.42 & -1.39 \\
3 & -1.57 & -1.57 & -1.48 & -1.43 & -1.37 & -1.33 & -1.30 & -1.27 & -1.25 & -1.21 \\
4 & -1.22 & -1.23 & -1.16 & -1.14 & -1.11 & -1.09 & -1.09 & -1.07 & -1.06 & -1.06 \\
5 & -1.03 & -1.00 & -0.97 & -0.95 & -0.93 & -0.92 & -0.90 & -0.89 & -0.88 & -0.88 \\
6 & -0.86 & -0.86 & -0.83 & -0.81 & -0.80 & -0.80 & -0.78 & -0.78 & -0.77 & -0.77 \\
7 & -0.77 & -0.76 & -0.74 & -0.73 & -0.72 & -0.70 & -0.70 & -0.69 & -0.69 & -0.69 \\
8 & -0.70 & -0.70 & -0.67 & -0.65 & -0.65 & -0.64 & -0.64 & -0.63 & -0.63 & -0.63 \\
9 & -0.65 & -0.64 & -0.61 & -0.60 & -0.60 & -0.59 & -0.59 & -0.58 & -0.58 & -0.58 \\
10 & -0.61 & -0.61 & -0.57 & -0.56 & -0.56 & -0.55 & -0.55 & -0.54 & -0.54 & -0.54
\end{tabular}

Table 12: Intercept $a_{w, \phi}$ (see Eq. (9)) of the linear regression of $\epsilon_{w, \phi}\left(0, f_{s} / 2.5\right)$ data points

\begin{tabular}{c|cccccccccc} 
& \multicolumn{10}{|c}{$\alpha_{w}$} \\
$\beta_{w}$ & 1 & 2 & 3 & 4 & 5 & 6 & 7 & 8 & 9 & 10 \\
\hline 2 & -27.7 & -14.5 & -17.2 & -18.5 & -21.4 & -20.9 & -22.5 & -23.1 & -23.1 & -23.1 \\
3 & -23.8 & -21.5 & -21.3 & -20.9 & -21.1 & -21.2 & -21.4 & -21.3 & -21.3 & -21.8 \\
4 & -24.4 & -23.1 & -23.2 & -23.0 & -22.8 & -22.9 & -22.6 & -22.6 & -22.5 & -22.2 \\
5 & -24.6 & -24.5 & -24.5 & -24.4 & -24.4 & -24.3 & -24.4 & -24.3 & -24.3 & -24.2 \\
6 & -25.8 & -25.3 & -25.5 & -25.7 & -25.5 & -25.3 & -25.5 & -25.3 & -25.4 & -25.4 \\
7 & -26.2 & -25.8 & -26.1 & -26.1 & -26.1 & -26.3 & -26.2 & -26.1 & -26.1 & -26.1 \\
8 & -26.5 & -26.3 & -26.7 & -26.9 & -26.8 & -26.8 & -26.8 & -26.8 & -26.8 & -26.7 \\
9 & -26.8 & -26.7 & -27.2 & -27.2 & -27.2 & -27.4 & -27.3 & -27.2 & -27.2 & -27.2 \\
10 & -27.0 & -26.8 & -27.6 & -27.5 & -27.5 & -27.6 & -27.6 & -27.7 & -27.6 & -27.6
\end{tabular}

15 of 18 
$O B_{w}=2 \quad \forall B_{w}=3 \quad \diamond B_{w}=4 \quad \nabla B_{w}=5 \quad \triangle B_{w}=6 \quad \forall B_{w}=7 \quad \triangleright B_{w}=8 \quad \square B_{w}=9$ \& $B_{w}=10$
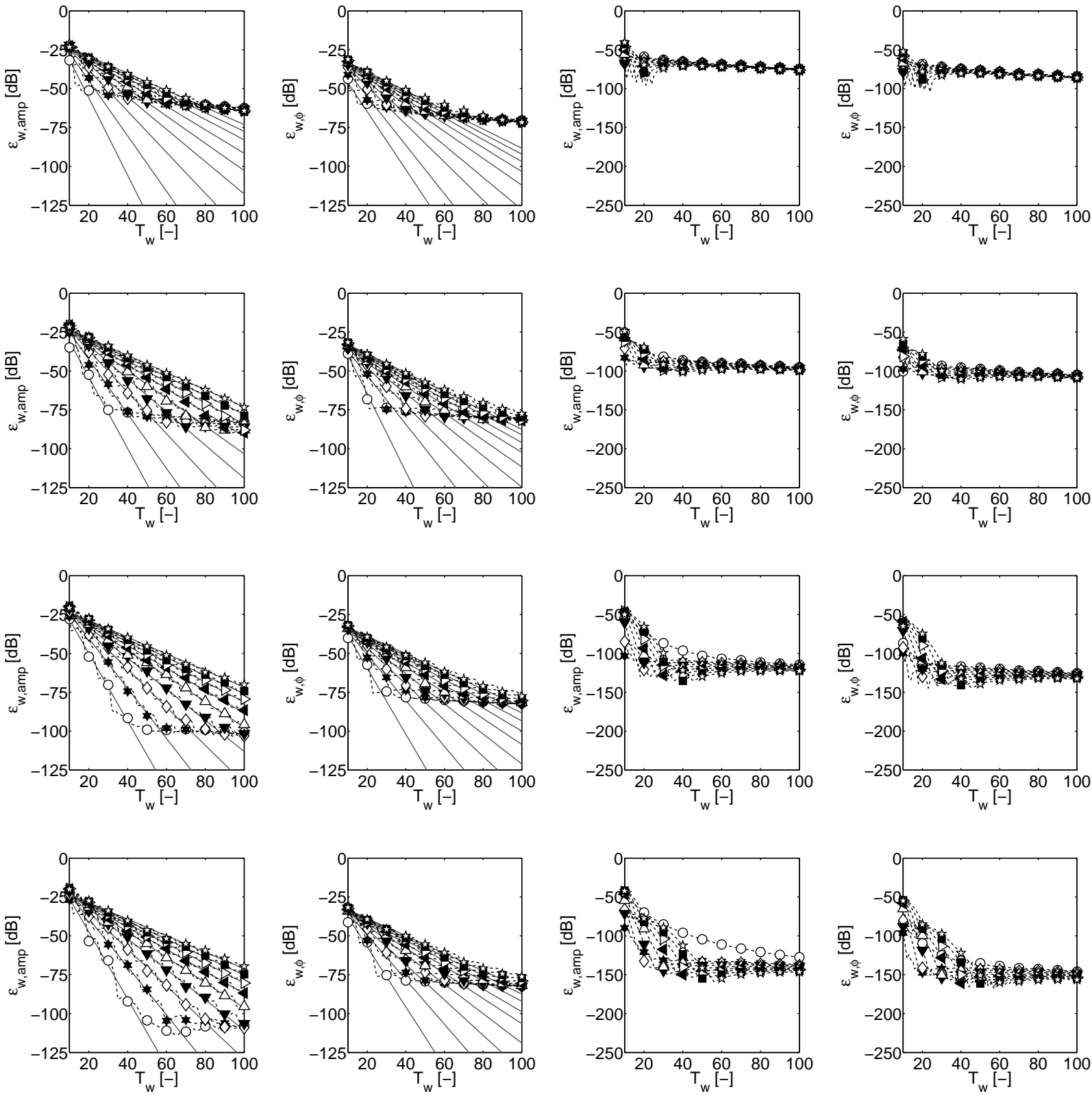

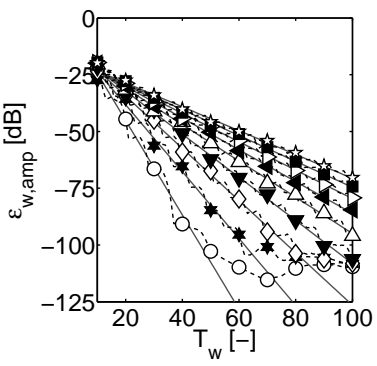

(a)

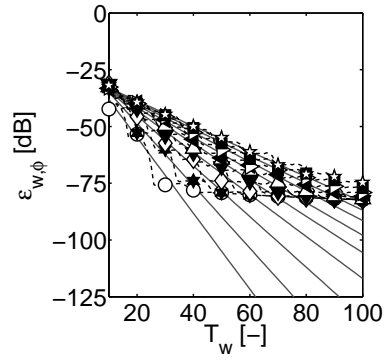

(b)

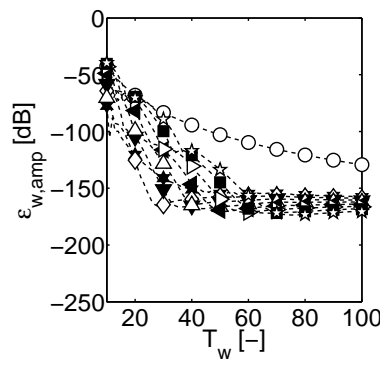

(c)

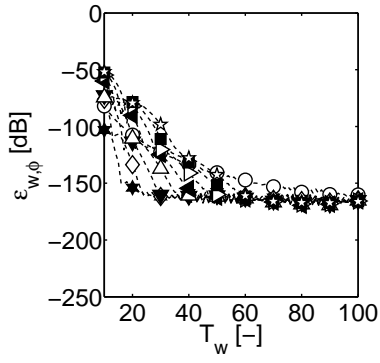

(d)

Figure 9: Error from the coupling DG to Fourier PSTD: (a) $\epsilon_{w, a m p}\left(0, f_{s} / 2.5\right)$, (b) $\epsilon_{w, \phi}\left(0, f_{s} / 2.5\right)$, (c) $\epsilon_{w, a m p}\left(0, f_{s} / 64\right)$ and $(\mathrm{d}) \epsilon_{w, \phi}\left(0, f_{s} / 64\right)$ (-- and marker). Columns (a) and (b) include the linear regression of data points (-). The errors are shown for $\alpha_{w}=1, \alpha_{w}=2, \alpha_{w}=3, \alpha_{w}=4, \alpha_{w}=5$, corresponding with row of graphs one to five, respectively. 

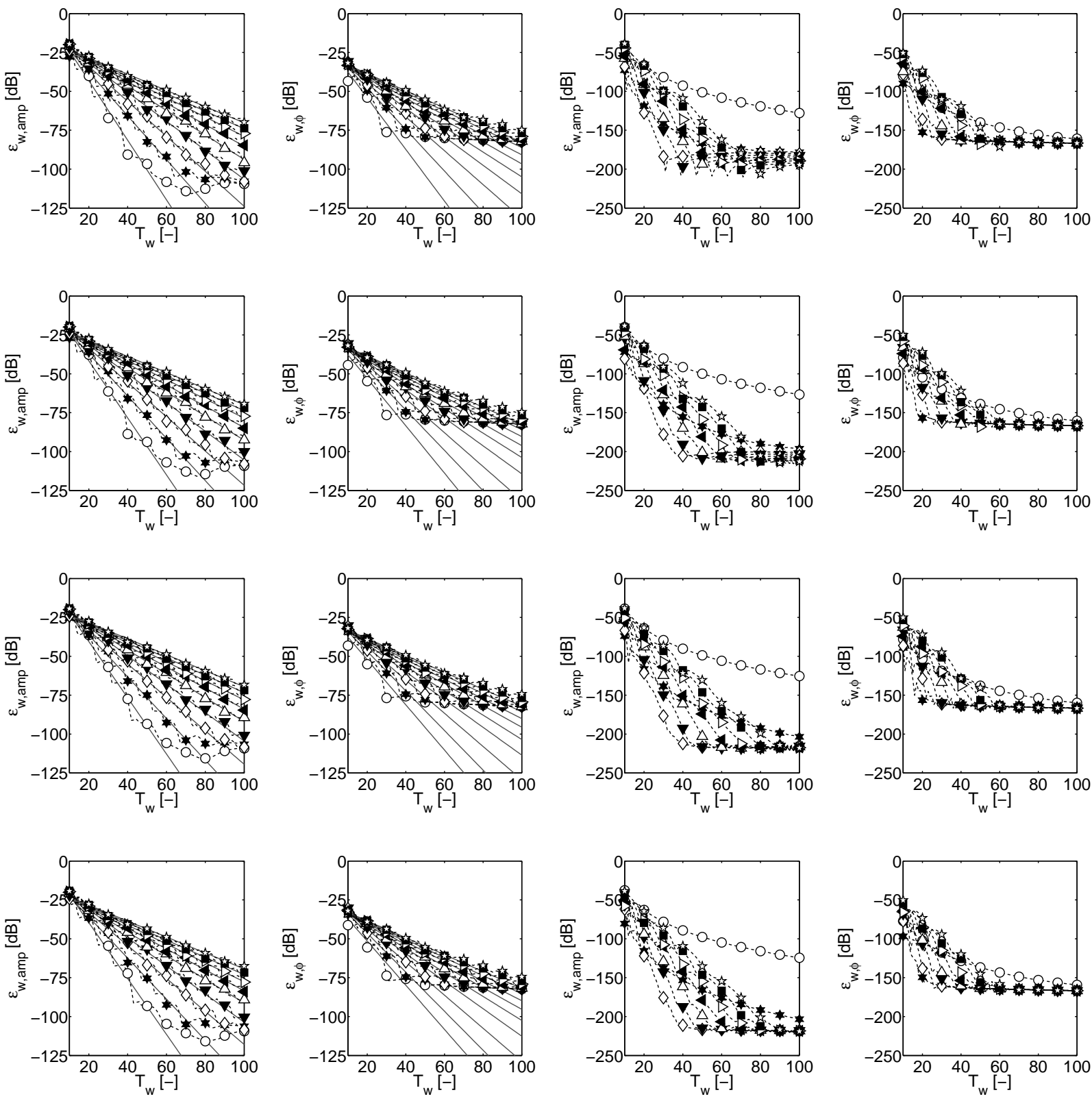

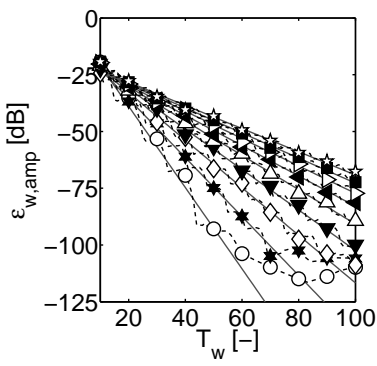

(a)

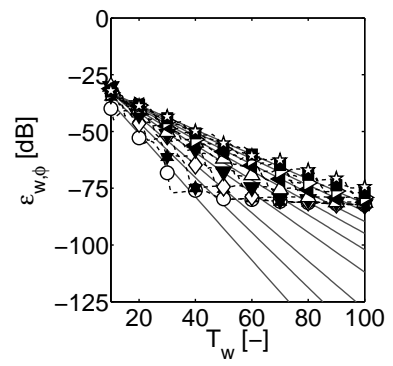

(b)

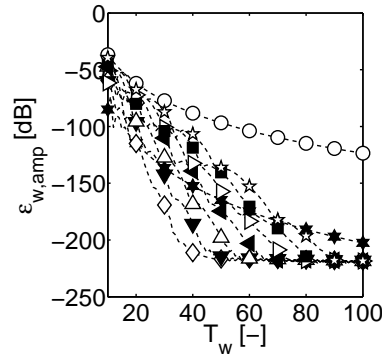

(c)

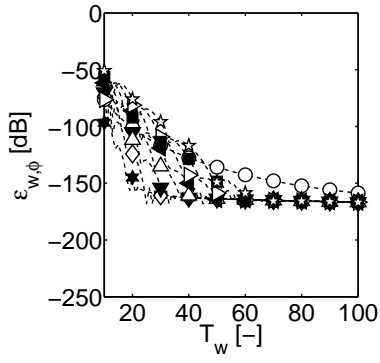

(d)

Figure 10: Error from the coupling DG to Fourier PSTD: (a) $\epsilon_{w, a m p}\left(0, f_{s} / 2.5\right)$, (b) $\epsilon_{w, \phi}\left(0, f_{s} / 2.5\right)$, (c) $\epsilon_{w, a m p}\left(0, f_{s} / 64\right)$ and $(\mathrm{d}) \epsilon_{w, \phi}\left(0, f_{s} / 64\right)$ (-- and marker). Columns (a) and (b) include the linear regression of data points (-). The errors are shown for $\alpha_{w}=6, \alpha_{w}=7, \alpha_{w}=8, \alpha_{w}=9, \alpha_{w}=10$, corresponding with row of graphs one to five, respectively. 


\section{Acknowledgments}

The research leading to these results has received funding from the People Programme (Marie Curie Actions) of the European Union's Seventh Framework Programme FP7/2007-2013 under REA grant agreement 290110, SONORUS "Urban Sound Planner".

\section{References}

\footnotetext{
${ }^{1}$ Hesthaven, J. S., Gottlieb, S. and Gottlieb, D., "Spectral Methods for Time-Dependent Problems," Cambridge: Cambridge University Press, 2007.

${ }^{2}$ Hornikx, M., Waxler, R. and Forssén, J., "The extended Fourier pseudospectral time-domain method for atmospheric sound propagation," The Journal of the Acoustical Society of America, vol. 128, October 2010, pp. 1632-1646.

${ }^{3}$ Hornikx, M., De Roeck, W., Toulorge, T. and Desmet, W., "Flow and geometrical effects on radiated noise from exhaust pipes computed by the Fourier pseudospectral time-domain method," Computers 83 Fluids, vol. 116, August 2015, pp. $176-191$.

${ }^{4} \mathrm{Liu}, \mathrm{Q} . \mathrm{H} .$, "The PSTD algorithm: A time-domain method requiring only two cells per wavelength," Microwave and Optical Technology Letters, vol. 15, June 1997, pp. 158-165.

${ }^{5}$ Liu, Q. H., "The pseudospectral time-domain (PSTD) algorithm for acoustic waves in absorptive media," IEEE transactions on ultrasonics, ferroelectrics, and frequency control, vol. 45, January 1998, pp. 1044-55.

${ }^{6} \mathrm{Spa}, \mathrm{C}$., Escolano, J. and Garriga, A., "Semi-empirical boundary conditions for the linearized acoustic Euler equations using Pseudo-Spectral Time-Domain methods," Applied Acoustics, vol. 72, March 2011, pp. 226-230.

${ }^{7}$ Van Renterghem, T., "Efficient outdoor sound propagation modeling with the finite-difference time-domain (FDTD) method: a review," International Journal of Aeroacoustics, vol. 13, October 2014, pp. 385-404.

${ }^{8}$ Hornikx, M., De Roeck, W. and Desmet, W., "A multi-domain Fourier pseudospectral time-domain method for the linearized Euler equations," Journal of Computational Physics, vol. 231, May 2012, pp. 4759-4774.

${ }^{9}$ Utzmann, J., Schwartzkopff, T., Dumbser, M. and Munz, C.-D., "Heterogeneous Domain Decomposition for Numerical Aeroacoustics," Multifield Problems in Solid and Fluid Mechanics SE - 13, R. Helmig, A. Mielke, and B. Wohlmuth, eds., Springer Berlin Heidelberg, 2006, pp. 429-459.

${ }^{10}$ Léger, R., Peyret, C. and Piperno, S., "Coupled Discontinuous Galerkin/Finite Difference Solver on Hybrid Meshes for Computational Aeroacoustics, " AIAA Journal, vol. 50, February 2012, pp. 338-349.

${ }^{11}$ Lisitsa, V., Tcheverda, V. and Botter, C., "Combination of the discontinuous Galerkin method with finite differences for simulation of seismic wave propagation," Journal of Computational Physics, vol. 311, April 2016, pp. 142-157.

${ }^{12}$ Dumbser, M. and Munz, C.-D., "ADER discontinuous Galerkin schemes for aeroacoustics," Comptes Rendus Mécanique, vol. 333, September 2005, pp. 683-687.

${ }^{13}$ Y. Reymen, W. De Roeck, G. Rubio, M. Baelmans and W. Desmet, "A 3D Discontinuous Galerkin Method for Aeroacoustic Propagation," Proceedings of the 12th International Congress on Sound and Vibration, Paper 387, July 2005.

${ }^{14}$ Toulorge, T., and Desmet, W., "Optimal RungeKutta schemes for discontinuous Galerkin space discretizations applied to wave propagation problems," Journal of Computational Physics, vol. 231, February 2012, pp. 2067-2091.

${ }^{15}$ Toulorge, T. and Desmet, W., "Curved Boundary Treatments for the Discontinuous Galerkin Method Applied to Aeroacoustic Propagation," AIAA Journal, April 2012.

${ }^{16}$ Atkins, H. L. and Chi-Shu, W., "Quadrature-free implementation of Discontinuous Galerkin method for hyperbolic equations," AIAA Journal, Vol. 36, No. 5, 1998, pp. 775-782.

${ }^{17}$ Hesthaven, J. S. and Warburton, T., "Nodal discontinuous Galerkin methods: Algorithms, analysis and applications," vol. 54, New York, NY: Springer New York, 2008.

${ }^{18}$ Williamson, J., "Low-storage Runge-Kutta schemes," Journal of Computational Physics, vol. 35, March 1980, pp. 48-56.

${ }^{19}$ Reymen, Y., Baelmans, M. and Desmet, W., "Efficient Implementation of Tam and Auriault's Time-Domain Impedance Boundary Condition," AIAA Journal, vol. 46, September 2008, pp. 2368-2376.

${ }^{20}$ Bogey, C. and Bailly, C., "A family of low dispersive and low dissipative explicit schemes for flow and noise computations," Journal of Computational Physics, vol. 194, February 2004, pp. 194-214.

${ }^{21} \mathrm{Hu}$, F. Q. and Atkins, H. L., "Eigensolution Analysis of the Discontinuous Galerkin Method with Nonuniform Grids," Journal of Computational Physics, vol. 182, November 2002, pp. 516-545.
} 